\title{
Dispersal Delays, Predator-Prey Stability, and the Paradox of Enrichment
}

\author{
Petra Klepac, ${ }^{a}$ Michael G. Neubert, ${ }^{a}$ P. van den Driessche ${ }^{b}$ \\ ${ }^{a}$ Biology Department, MS \#34, Woods Hole Oceanographic Institution, Woods \\ Hole, MA 02543-1049, USA \\ ${ }^{\mathrm{b}}$ Department of Mathematics and Statistics, University of Victoria, Victoria, \\ British Columbia, V8W 3P4, Canada
}

\begin{abstract}
It takes time for individuals to move from place to place. This travel time can be incorporated into metapopulation models via a delay in the interpatch migration term. Such a term has been shown to stabilize the positive equilibrium of the classical Lotka-Volterra predator-prey system with one species (either the predator or the prey) dispersing.

We study a more realistic, Rosenzweig-MacArthur, model that includes a carrying capacity for the prey, and saturating functional response for the predator. We show that dispersal delays can stabilize the predator-prey equilibrium point despite the presence of a Type II functional response that is known to be destabilizing. We also show that dispersal delays reduce the amplitude of oscillations when the equilibrium is unstable, and therefore may help resolve the paradox of enrichment.
\end{abstract}

Key words: delay-differential equations, distributed delay, MacArthur-Rosenzweig model, metapopulation dynamics, paradox of enrichment, stability

\section{Introduction}

The basic models of predator-prey and host-parasitoid systems predict unstable equilibria, often accompanied by large-amplitude oscillations in both species. These oscillations drive the populations to low densities, and have been interpreted as potential causes of extinction. In contrast, natural predator-prey systems seem to persist for long periods. Theoreticians and experimentalists

Email addresses: petra@whoi.edu (Petra Klepac,), mneubert@whoi.edu (Michael G. Neubert,), pvdd@math.uvic.ca (P. van den Driessche). 
have suggested a number of potential processes that might resolve this conflict between models and data (see, for example, May, 1973; Hassell, 1978; Crawley, 1992; Mueller and Joshi, 2000). Spatial processes, and in particular metapopulation structure, have garnered significant attention (Taylor, 1990; Briggs and Hoopes, 2004).

Dispersal, the process that distinguishes spatial models from their nonspatial counterparts, has been added to predator-prey models in many different ways, with varying effects on stability (Briggs and Hoopes, 2004). One way to include dispersal is to distinguish a class of dispersing individuals, that, while dispersing, do not participate in the predator-prey interaction. A number of authors have shown that including such a pool of dispersers (be they predators or prey) in a Lotka-Volterra model stabilizes coexistence at an equilibrium point. The models of Holt (1984), Weisser and Hassell (1996) and Weisser et al. (1997) include the dispersal pool explicitly, and couple it to the dynamics within a patch via constant per capita immigration and emigration rates. These models implicitly assume an exponential distribution of the time that an individual spends dispersing.

Exponential travel-time distributions, however, have some biological peculiarities. For example, there is no maximum travel time, and the modal traveltime is zero. To see if these implicit assumptions play a role in stabilizing the equilibrium, Neubert et al. (2002) relaxed this assumption by prescribing an arbitrary distribution of dispersal times. They showed that, except in cases so rare as to be biologically irrelevant, the stabilizing effect of such "dispersal delays" remains.

All of these analyses are based upon the Lotka-Volterra predator-prey model

$$
\begin{aligned}
& \frac{d N}{d T}=(R-A P) N \\
& \frac{d P}{d T}=(B N-M) P
\end{aligned}
$$

where $N$ is the population density of the prey and $P$ is the population density of the predator. The prey population has a constant per capita growth rate $R$, and the predator population has a constant per capita mortality rate $M$. The predator-prey interaction is captured by linear functional and numerical responses, scaled by the parameters $A$ and $B$. The parameters $R, A, B$, and $M$ are assumed to be positive.

Model (1) has a unique coexistence equilibrium point (i.e., an equilibrium point at which both species have positive densities) at $N=M / B, P=R / A$. This equilibrium point is a center, surrounded by a family of periodic orbits whose amplitudes depend on the initial population sizes. Adding either preda- 
tor or prey dispersal to this model stabilizes the equilibrium point if dispersal delays are accounted for (Neubert et al., 2002). In the absence of delays, predator dispersal reduces the amplitude of the oscillations but does not stabilize the equilibrium point (Jansen, 1995; Jansen and de Roos, 2000). Increasing the number of patches in this model gives rise to other equilibria in which the prey are absent from one or more patches (see, for example, Feng and Hinson, 2005) that we do not consider here.

Model (1), and its spatial extensions, have been criticized as being oversimplified for two reasons. First, in the absence of the predators, the prey grow exponentially without bound. Second, the per capita rate of consumption of prey by predators grows in proportion to the prey population size, implying that individual predators can process prey items infinitely fast. These faults are eliminated in the Rosenzweig-MacArthur model (Rosenzweig and MacArthur, 1963)

$$
\begin{aligned}
& \frac{d N}{d T}=R N\left(1-\frac{N}{K}\right)-\frac{A N P}{C+N} \\
& \frac{d P}{d T}=\frac{B N P}{C+N}-M P
\end{aligned}
$$

which includes a carrying capacity for the prey $(K)$ and a finite prey handling time for the predators that results in a saturating functional response. Here, $A$ is the maximum rate at which an individual predator can consume prey and $C$ is the prey density at which an individual predator's consumption rate equals $A / 2$. The ratio $B / A$ gives the fraction of consumed prey that are converted into predators.

The dynamics of model (2) are more complicated than those of model (1) (Kot, 2001). For small values of carrying capacity, the coexistence equilibrium point is locally asymptotically stable. As the carrying capacity increases beyond some threshold value, the equilibrium point becomes unstable, and trajectories are drawn onto a single stable limit cycle. The amplitude of predatorprey oscillations increases with increasing prey's carrying capacity, reaching vanishingly small densities at which natural populations cannot persist. This destabilization by increasing prey carrying capacity is known as the 'paradox of enrichment' (Rosenzweig, 1971; May, 1972; Gilpin, 1972).

Here we present three major findings. First, we show that dispersal delays can stabilize the coexistence equilibrium point of model (2) (as they did in model (1)) by delineating the stability region in parameter space. Second, we show that for many parameter values, stability persists in a so-called "Type II model" wherein prey growth is density independent (i. e. model (2) in the limit of infinite carrying capacity $K$ ). We thus establish that delayed dispersal can overcome a destabilizing Type II functional response even in the 
absence of stabilizing prey density-dependence. Finally, we show that dispersal delays help resolve the paradox of enrichment by reducing the amplitude of oscillations when the equilibrium is unstable, thereby preventing the small population sizes that might lead to extinction.

We begin, in the next section, by constructing a Rosenzweig-MacArthur model that incorporates dispersal delays. Using the methods outlined in Neubert et al. (2002), it can be shown that if dispersal delays stabilize the single patch model they also stabilize a spatially homogeneous equilibrium of a model with an arbitrary number of identical patches. Therefore, we limit our investigation to a single habitat patch from which only predators disperse. We then present results for two types of dispersal delay: a discrete delay that implies that all individuals spend exactly the same amount of time away from the patch, and a distributed delay that accounts for differences in, for example, dispersal ability between individuals. For discrete-delays our results are derived from numerical simulations. In the case of a distributed delay with Erlang distribution, we analytically derive a polynomial characteristic equation, whose roots we find numerically. We conclude with a brief discussion.

\section{Model}

The model that we analyze,

$$
\begin{aligned}
& \frac{d N}{d T}=R N\left(1-\frac{N}{K}\right)-\frac{A N P}{C+N} \\
& \frac{d P}{d T}=\frac{B N P}{C+N}-M P+D\left[\int_{0}^{\infty} G(S) \mathrm{e}^{-M_{P} S} P(T-S) d S-P\right]
\end{aligned}
$$

describes the dynamics of a sedentary prey and a mobile predator in a single habitat patch. Individual predators emigrate from the patch at the constant per capita rate $D$, and return $S$ units of time after their departure. ${ }^{1}$ To account for the differences in dispersal abilities between predators, we define a distribution of dispersal delays, $G(S) \geq 0$, for the time a predator takes to disperse, given that it survives the trip (Neubert et al., 2002). Because all dispersal times are nonnegative it follows that $\int_{0}^{\infty} G(S) d S=1$ (see also Azer and van den Driessche, 2006). We assume that the probability of surviving a trip of duration $S$ is $e^{-M_{p} S}$, where $M_{p}$ is the mortality rate during the migration.

Model (3) takes the form of a delay differential equation with distributed delay.

$\overline{1}$ For notational convenience, a variable with no time dependence explicitly given is to be evaluated at the current (undelayed) time. 
For examples of how such equations have been used in other types of ecological models, and for how they may be analyzed, see the books by Kuang (1993) and MacDonald (1989).

In order to reduce the number of parameters, and simplify our analyses, we scale the variables and parameters of the model (3) according to

$$
\begin{gathered}
t=R T, \quad s=R S, \quad \mu=M / R, \quad d=D / R, \quad \mu_{p}=M_{P} / R \\
p=A P / R C, \quad n=B N / R C, \quad \varepsilon=R / B, \quad \kappa=K B / R C .
\end{gathered}
$$

Substitution into system (3) gives the dimensionless form

$$
\begin{aligned}
& \dot{n}=n\left(1-\frac{n}{\kappa}\right)-\frac{n p}{1+\varepsilon n}, \\
& \dot{p}=\frac{n p}{1+\varepsilon n}-\mu p+d\left[\int_{0}^{\infty} g(s) \mathrm{e}^{-\mu_{p} s} p(t-s) d s-p\right],
\end{aligned}
$$

where $g(s)$ is the scaled version of $G(S)$.

In Sec. 3, we focus on the effects of $\kappa$ (the dimensionless carrying capacity) and $d$ (the dimensionless emigration rate) on the stability of the unique coexistence equilibrium for model (5):

$$
n^{*}=\frac{\mu+d\left(1-\tilde{g}\left(\mu_{p}\right)\right)}{1-\varepsilon\left[\mu+d\left(1-\tilde{g}\left(\mu_{p}\right)\right)\right]}, \quad p^{*}=\left(1-\frac{n^{*}}{\kappa}\right)\left(1+\varepsilon n^{*}\right) .
$$

Here, $\tilde{g}(x)$ is the (one-sided) Laplace transform of the travel-time distribution $g(s)$, i. e.,

$$
\tilde{g}(x) \equiv \int_{0}^{\infty} g(s) \mathrm{e}^{-x s} d s .
$$

The equilibrium (6) is positive only if

$$
d<\frac{\kappa(1-\varepsilon \mu)-\mu}{(\kappa \varepsilon+1)\left[1-\tilde{g}\left(\mu_{p}\right)\right]} .
$$

If $d$ is too large, and inequality (8) is violated, the predators do not spend sufficient time feeding on the prey patch to maintain a positive growth rate and are extirpated as a result.

To determine the stability of the coexistence equilibrium point (6) of model (5) we must determine the fate of small perturbations, $u(t)$ and $v(t)$, to the coexistence equilibrium. Set

$$
n(t)=n^{*}+u(t), \quad p(t)=p^{*}+v(t) .
$$

For $|u|$ and $|v|$ sufficiently small, the dynamics of these perturbations are approximated by the linear system 


$$
\begin{aligned}
\dot{u}= & u\left[1-\frac{2 n^{*}}{\kappa}-\frac{p^{*}}{\left(1+\varepsilon n^{*}\right)^{2}}\right]+v\left(-\frac{n^{*}}{1+\varepsilon n^{*}}\right), \\
\dot{v}= & u\left(\frac{p^{*}}{1+\varepsilon n^{*}}\right)+v\left(\frac{n^{*}}{1+\varepsilon n^{*}}-\mu-d\right) \\
& +d \int_{0}^{\infty} g(\tau) \mathrm{e}^{-\mu_{p} \tau} v(t-\tau) d \tau .
\end{aligned}
$$

Looking for solutions to (10) of the form

$$
\left(\begin{array}{l}
u \\
v
\end{array}\right)=\mathbf{w ~}^{\lambda t}, \quad \mathbf{w} \neq 0
$$

we find that $\lambda$ and $\mathbf{w}$ must satisfy

$$
(\mathbf{J}-\lambda \mathbf{I}) \mathbf{w}=0
$$

where $\mathbf{J}$ is the Jacobian matrix

$$
\mathbf{J}=\left[\begin{array}{cc}
1-\frac{2 n^{*}}{\kappa}-\frac{p^{*}}{\left(1+\varepsilon n^{*}\right)^{2}} & -\frac{n^{*}}{1+\varepsilon n^{*}} \\
\frac{p^{*}}{\left(1+\varepsilon n^{*}\right)^{2}} & d\left[\tilde{g}\left(\mu_{p}+\lambda\right)-\tilde{g}\left(\mu_{p}\right)\right]
\end{array}\right]
$$

Equation (12) has solutions with $\mathbf{w} \neq 0$ only if $\operatorname{det}(\mathbf{J}-\lambda \mathbf{I})=0$, which translates to

$$
H(\lambda)=K(\lambda)
$$

with

$$
\begin{aligned}
& H(\lambda)=\left[\lambda+\frac{2 n^{*}}{\kappa}+\frac{p^{*}}{\left(1+\varepsilon n^{*}\right)^{2}}-1\right]\left[\lambda+d \tilde{g}\left(\mu_{p}\right)\right]+\frac{n^{*} p^{*}}{\left(1+\varepsilon n^{*}\right)^{3}}, \\
& K(\lambda)=\left[\lambda+\frac{2 n^{*}}{\kappa}+\frac{p^{*}}{\left(1+\varepsilon n^{*}\right)^{2}}-1\right] d \tilde{g}\left(\mu_{p}+\lambda\right) .
\end{aligned}
$$

The roots of this "characteristic" equation are the eigenvalues; they are, in general, complex numbers.

The real parts of the eigenvalues determine the stability of the equilibrium point. If all of the eigenvalues have negative real parts, $u$ and $v$ will vanish in the limit $t \rightarrow \infty$, and the equilibrium point is therefore locally stable. If any eigenvalue has a positive real part, the perturbations grow, and the equilibrium is unstable. Note that (14) and (15) together imply that $\lambda=0$ is not an eigenvalue since $n^{*}$ and $p^{*}$ are positive.

In the absence of dispersal, $d=0$. In this case, local stability of the equilibrium 
point is guaranteed from (8) and (14) if

$$
\frac{\mu}{1-\varepsilon \mu}<\kappa<\frac{1+\varepsilon \mu}{\varepsilon(1-\varepsilon \mu)}
$$

For finite $\kappa$, if the left-hand inequality is violated the predator is extirpated, since for this parameter range there is no positive steady state; see inequality (8). Violation of the right-hand inequality results in a Hopf bifurcation and a predator-prey limit cycle (Kot, 2001). Note that in the limit $\kappa \rightarrow \infty$, the equilibrium point is never stable.

\section{Results}

\subsection{Discrete travel time}

If the duration of every dispersal event of every individual is exactly $\tau$, then the dispersal delay distribution is a delta function: $g(s)=\delta(s-\tau)$ and $\tilde{g}(x)=\exp (-\tau x)$. We have been unable to analytically infer the local stability of the coexistence equilibrium in this case, as the characteristic equation (14) is a transcendental equation with infinitely many solutions. Therefore, we illustrate our results (in Fig. 1) using numerically generated stability diagrams in the $(\tau, d)$ parameter plane for various values of $\kappa$. For each combination of the parameters, we (i) calculated the equilibrium point (6), (ii) for coexistence equilibria we chose a random initial condition for the prey and the predator uniformly distributed between $50 \%$ and $150 \%$ of the equilibrium values, (iii) using the Simulink package in Matlab, we simulated the model (5) and discarded the transient dynamics. We then distinguished three sets in $(\tau, d)$ parameter plane: (a) a set of parameters for which the coexistence equilibrium does not exist (because inequality (8) is violated), (b) a set for which the coexistence equilibrium exists but it is unstable, and (c) a set for which the coexistence equilibrium is stable.

We start (Figure 1A) with a case that is stable in the absence of the dispersal delay (i.e., satisfying (16)). As expected, the coexistence equilibrium is stable everywhere it exists. In Figures $1 \mathrm{~B}-\mathrm{D}$ the values of $\kappa$ violate the right-most inequality in (16). For these values of the carrying capacity, the equilibrium point of the Rosenzweig-MacArthur model (2) is unstable, surrounded by a predator-prey cycle. Dispersal delay dampens the predator-prey oscillation resulting in the area of stable equilibrium shown in white. For $\kappa \gtrsim 1000$, the stability region reduces to four "islands".

Since stability diagrams do not reveal the details of the unstable behavior, we generated bifurcation diagrams for different values of parameters, for both 
predator and prey densities. All diagrams exhibit qualitatively similar behavior, so we show only one bifurcation diagram for prey density with $\tau$ as the bifurcation parameter (Fig. 2).

For each value of $\tau$ we simulated model (5), discarded the transient dynamics, and present only the final behavior by plotting only the local maxima and minima of the trajectory. Stable equilibria therefore appear as a single point. Oscillations with one peak appear as two points, and oscillations with two peaks appear as four points, et cetera. Quasi-periodic and aperiodic oscillations appear as "smears."

In addition to quasi-periodic and aperiodic behavior, the bifurcation diagrams also reveal the coexistence of multiple attractors. In Fig. 2A, we increased $\tau$ from 0 to 7 in small steps, using the end of the simulation for one value of $\tau$ as the initial condition of the simulation for the following value of $\tau$. We followed the same procedure in Fig. 2B, except that we decreased $\tau$ from 7 to 0 . For values of $\tau$ in the shaded regions of Fig. 2, solutions converge to different attractors depending on initial conditions.

In Fig. 3 we categorize the dynamics of the Type II model,

$$
\begin{aligned}
& \dot{n}=n-\frac{n p}{1+\varepsilon n}, \\
& \dot{p}=\frac{n p}{1+\varepsilon n}-\mu p+d\left[\int_{0}^{\infty} g(s) \mathrm{e}^{-\mu_{p} s} p(t-s) d s-p\right],
\end{aligned}
$$

in the $(\tau, d)$ parameter space over a range of $\varepsilon$. In Fig. 3A, the predator's functional response is strong ( $\varepsilon$ is relatively large) and the equilibrium cannot be stabilized by dispersal delays. As $\varepsilon$ decreases, however, stable islands grow in number and in size. In the limit $\varepsilon \rightarrow 0$, Neubert et al. (2002) showed the equilibrium is stable everywhere except for a set of measure zero in the $(\tau, d)$ plane. Comparing Fig. 3C with Fig. 1D shows that the stability properties of the Type II model are essentially the same as the Rosenzweig-MacArthur model with large carrying capacity.

The stability in the Type II model implies that dispersal delays can help resolve the paradox of enrichment. In the MacArthur-Rosenzweig model without dispersal, the amplitude of oscillation increases with increasing carrying capacity and the population soon reaches vanishingly small densities. We illustrate this with a bifurcation diagram with $\kappa$ as a bifurcation parameter (Fig. 4A, C). We again present only the long-term dynamics by plotting local minima and maxima of the trajectory. The minimal population density decreases rapidly with increasing $\kappa$ and eventually becomes dominated by numerical round-off errors, so the graphs in Fig. 4A and $\mathrm{C}$ appear blurred. For comparison, in Fig. $4 \mathrm{~B}$ and $\mathrm{D}$ we show how the amplitude of the oscillation changes with increasing capacity in the presence of discrete dispersal delays. In this case, 
large values of $\kappa$ give rise to quasi-periodic and aperiodic behavior, but minimal population densities remain well above zero for both prey (Fig. 4B) and predator (Fig. 4D).

\subsection{Distributed travel time}

When the movement abilities of the predators differ, or the vagaries of dispersal affect individuals differently, individual travel-times form some distribution. For mathematical convenience we study a case where the delay distribution is an Erlang distribution

$$
g(s)=g_{b, c}(s)=\frac{b^{c} s^{c-1} \mathrm{e}^{-b s}}{(c-1) !}
$$

with shape parameter $c$ and scale parameter $b$ (Fig. 5). For $c=1$ the distribution is exponential, and the dispersal model is equivalent to one that includes an explicit pool of dispersers with constant per capita emigration and immigration rates, à la Weisser and Hassell (1996) and Weisser et al. (1997). For $c>1$ the mode of the distribution, at $(c-1) / b$, is positive. For large $c$, the mode approaches the mean, $\tau_{a v}=c / b$, and the distribution resembles a delta function.

For this special family of distributions, we can determine the local stability of the equilibrium point (6) by linearizing system (5) and using the Laplace transform

$$
\tilde{g}(x)=\tilde{g}_{b, c}(x)=\frac{b^{c}}{(x+b)^{c}} .
$$

The characteristic equation (14) then reduces to a polynomial of degree $c+2$ :

$$
\left[(\lambda+\mu+d)\left(\lambda p^{*}-\varepsilon n^{*}\right)+n^{*}(1-\lambda)\right]\left(\frac{b+\mu_{p}+\lambda}{b}\right)^{c}=d\left(\lambda p^{*}-\varepsilon n^{*}\right) .
$$

To construct the stability diagrams in Fig. 6, we found the roots of equation (20) numerically using the Matlab function $\operatorname{roots}($ ).

In Figs. 6A-C we show stability diagrams for increasing values of the shape parameter $c$, with the other parameters fixed at levels that produce an unstable equilibrium in the absence of dispersal delays.

The area in the parameter space where the equilibrium point is stable is largest for $c=1$ (Fig. 6A), implying that the stabilizing effect of dispersal delays is strongest when individual travel times are exponentially distributed. As $c$ increases, the stability region shrinks and its borders become more convoluted. The area of stability remains large even in the limit as $\kappa \rightarrow \infty$ (Fig. 6D). We expect that as $c$ becomes even larger the stability diagram would look 
even more like the discrete-delay case. Unfortunately, for $c$ much larger than 64, the characteristic polynomial (20) is extremely poorly conditioned, with coefficients differing in magnitude by hundreds of orders; we have been unable to construct a stability diagram for these cases.

\section{Discussion}

We have shown that the coexistence equilibrium point of the single-patch Rosenzweig-MacArthur model (2) can be stabilized when predator dispersal includes a dispersal delay. Stabilization occurs because the dispersal delays introduce density dependence into the dispersal process (Murdoch et al., 1992; Neubert et al., 2002). If the predator population on the patch is abundant compared to earlier times, emigration from the patch will exceed immigration, and the abundance on the patch decreases. If, on the other hand, the current population on the patch is small, immigration will exceed emigration, thereby increasing the population size. In this way, population oscillations are reduced and species abundances eventually reach their equilibrium levels.

The stabilizing effect of a dispersal delay is strongest when the individual travel times are exponentially distributed, as they are in models that include a pool of dispersers. The stabilizing effect weakens as the delay distribution becomes more concentrated around its mode. In the weakest case, when the delay distribution is a delta function and the carrying capacity is infinite, the stability region takes the shape of an archipelago (Fig. 3B-D). The same structure, dubbed "islands of amplitude death," has been observed in mathematical studies of coupled oscillators (Reddy et al., 1998, 1999). These studies find that the amplitude of two coupled limit-cycle oscillators can be "quenched" when the coupling is time delayed.

Dispersal delays are less effective at stabilizing the equilibrium as the carrying capacity of the prey increases. Nevertheless, for a significant set of parameter values, the model with dispersal delays has a stable coexistence equilibrium even for an infinite carrying capacity (see Fig. 3 and Fig. 6D). Thus dispersal delays alone are capable of inducing stability in the face of a destabilizing Type II functional response.

Even when the equilibrium is unstable, the amplitude of the predator-prey oscillation does not grow with increasing carrying capacity, and the minimum population densities remain well above zero (Fig. 4B, and D). In this sense, our results can be added to those of Jansen (1995) (see also de Roos et al., 1991; Scheffer and de Boer, 1995; Nisbet et al., 1998; Jansen and de Roos, 2000; Jansen, 2001) who also proposed dispersal (without delay) as a potential resolution of the paradox of enrichment. The stabilizing effect of dispersal in 
these studies is weaker than it is in our model, however, as it only produces a decrease in the amplitude of the limit cycle, rather than stabilizing the equilibrium point.

Spatial structure is by no means the only factor that has been proposed to resolve the paradox (Abrams and Walters, 1996). Other factors include heterogeneity within the prey population and complex food web structure. Enrichment of the prey can reduce the amplitude of population cycles when prey have different profitability (Genkai-Kato and Yamamura, 1999) or when a single predator attacks two prey species, one of which is inedible (Kretzschmar et al., 1993). Enrichment can even lead to stability in systems that have a prey refuge (Abrams and Walters, 1996; Gurney and Veitch, 2000) or inducible defences in prey (Vos et al., 2004). Enhanced system persistence and stability in intricate food webs has been attributed to weak trophic interactions that dampen oscillations between consumers and resources and maintain population densities further away from zero (McCann et al., 1998).

Our analysis has several limitations. We focussed on a single habitat patch from which only predators dispersed. Using methods outlined Neubert et al. (2002), one can show that if dispersal delays stabilize the single patch model they also stabilize a spatially homogeneous equilibrium of a model with an arbitrary number of identical patches. Many real metapopulations, however, are composed of numerous patches that differ in several attributes. In particular, the distance between two patches, and therefore the distribution of dispersal delays between them, will not be the same for all pairs of patches. Furthermore, both prey and predators may disperse. Our analysis does not apply to these more complicated scenarios.

Finally, we note that our results may depend upon the exact way in which we modeled the dispersal process. Another approach uses so called "patch occupancy models," which keep track of the number of habitat patches that are in various states, e.g., empty, or occupied by prey, or occupied by predators. In contrast to our results, Sabelis et al. (1991) showed that while the addition of a pool of dispersing prey was stabilizing in a simple patch occupancy model, dispersing predators could be destabilizing. When it comes to the effects of dispersal on predator-prey dynamics, the details of how dispersal is incorporated appear to be important.

\section{Acknowledgements}

Discussions with Bard Ermentrout and suggestions from Will Wilson and three anonymous reviewers improved the presentation of the model and numerical analysis. MGN and PK were supported by grants from the National Science 
Foundation (OCE-0083976, DEB-0235692, DEB-9973212) and Environmental Protection Agency (R-82908901-0). The research of PvdD is partially supported by NSERC and MITACS.

\section{References}

Abrams, P. A., Walters, C. J., 1996. Invulnerable prey and the paradox of enrichment. Ecology 77, 1125-1133.

Azer, N., van den Driessche, P., 2006. Competition and dispersal in patchy environments. Mathematical Biosciences and Engineering 3 (2), 283-296.

Briggs, C. J., Hoopes, M. F., 2004. Stabilizing effects in spatial parasitoid-host and predator-prey models: a review. Theor. Pop. Biol. 65, 299-315.

Crawley, M. J., 1992. Natural Enemies: The Population Biology of Predators, Parasites and Diseases. Blackwell Scientific, London.

de Roos, A. M., McCauley, E., Wilson, W. G., 1991. Mobility versus densitylimited predator-prey dynamics on different spatial scales. Proc. R. Soc. Lond. B 246, 117-122.

Feng, W., Hinson, J., 2005. Stability and pattern for two-patch predatorprey population dynamics. Discrete and Continuous Dynamical Systems Supplement Volume 2005, 268-279.

Genkai-Kato, M., Yamamura, N., 1999. Unpalatable prey resolves the paradox of enrichment. Proc. R. Soc. Lond. B 266, 1215-1219.

Gilpin, M. E., 1972. Enriched predator-prey systems: Theoretical stability. Science 177, 902-904.

Gurney, W. S. C., Veitch, A. R., 2000. Self-organization, scale and stability in a spatial predator-prey interaction. Bull. Math. Biol. 62, 61-86.

Hassell, M. P., 1978. The Dynamics of Arthropod Predator-Prey Systems. Princeton University Press, Princeton, NJ.

Holt, R. D., 1984. Spatial heterogeneity, indirect interactions, and the coexistence of prey species. Am. Nat. 124, 377-406.

Jansen, V. A. A., 1995. Regulation of predator-prey systems through spatial interactions: a possible solution to the paradox of enrichment. Oikos 74, 384-390.

Jansen, V. A. A., 2001. The dynamics of two diffusively coupled predator-prey populations. Theor. Pop. Biol. 59, 119-131.

Jansen, V. A. A., de Roos, A. M., 2000. The Geometry of Ecological Interactions: Simplifying Spatial Complexity. Cambridge University Press, Cambridge, U.K., Ch. The role of space in reducing predator-prey cycles, pp. $183-201$.

Kot, M., 2001. Elements of Mathematical Ecology. Cambridge University Press, Cambridge, UK.

Kretzschmar, M., Nisbet, R. M., McCauley, E., 1993. A predator-prey model for zooplankton grazing on competing algal populations. Theor. Pop. Biol. 
44, 32-66.

Kuang, Y., 1993. Delay Differential Equations with Applications in Population Dynamics. Academic Press, Boston.

MacDonald, N., 1989. Biological Delay Systems: Linear Stability Theory. Cambridge University Press, Princeton, NJ.

May, R. M., 1972. Limit cycles in predator-prey communities. Science 177, 900-902.

May, R. M., 1973. Stability and Complexity in Model Ecosystems. Princeton University Press, Princeton, NJ.

McCann, K., Hastings, A., Huxel, G. R., 1998. Weak trophic interactions and the balance of nature. Nature 395, 794-798.

Mueller, L. D., Joshi, A., 2000. Stability in Model Populations. Princeton University Press, Princeton, NJ.

Murdoch, W. W., Briggs, C. J., Nisbet, R. M., Gurney, W. S. C., StewartOaten, A., 1992. Aggregation and stability in metapopulation models. Am. Nat. 140, 41-58.

Neubert, M. G., Klepac, P., van den Driessche, P., 2002. Stabilizing dispersal delays in predator-prey metapopulation models. Theor. Pop. Biol. 61, 339347.

Nisbet, R. M., de Roos, A. M., Wilson, W. G., Snyder, R., 1998. Discrete consumers, small scale resource heterogeneity, and population stability. Ecology Letters 1, 34-37.

Reddy, D. V. R., Sen, A., Johnson, G. L., 1998. Time delay induced death in coupled limit cycle oscillators. Phys. Rev. Lett. 80, 5109-5112.

Reddy, D. V. R., Sen, A., Johnson, G. L., 1999. Time delay effects in coupled limit cycle oscillators at Hopf bifurcation. Physica D 129, 15-34.

Rosenzweig, M. L., 1971. Paradox of enrichment: destabilization of exploitation ecosystems in ecological time. Science 171, 285-387.

Rosenzweig, M. L., MacArthur, R. H., 1963. Graphical representation and stability conditions of predator-prey interactions. Am. Nat. 97, 209-223.

Sabelis, M. W., Diekmann, O., Jansen, V. A. A., 1991. Metapopulation persistence despite local extinction: predator-prey patch models of the LotkaVolterra type. Biol. J. Linn. Soc. 42, 267-283.

Scheffer, M., de Boer, R. J., 1995. Implications of spatial heterogeneity for the paradox of enrichment. Ecology 76, 2270-2277.

Taylor, A. D., 1990. Metapopulations, dispersal, and predator-prey dynamics: an overview. Ecology 71, 429-433.

Vos, M., Kooi, B. W., DeAngelis, D. L., Mooij, W. M., 2004. Inducible defences and the paradox of enrichment. Oikos 105, 471-480.

Weisser, W. W., Hassell, M. P., 1996. Animals on the move stabilize hostparasitoid systems. Proc. R. Soc. Lond. B 263, 749-754.

Weisser, W. W., Jansen, V. A. A., Hassell, M. P., 1997. The effects of a pool of dispersers on host-parasitoid systems. J. Theor. Biol. 189, 413-425. 
A
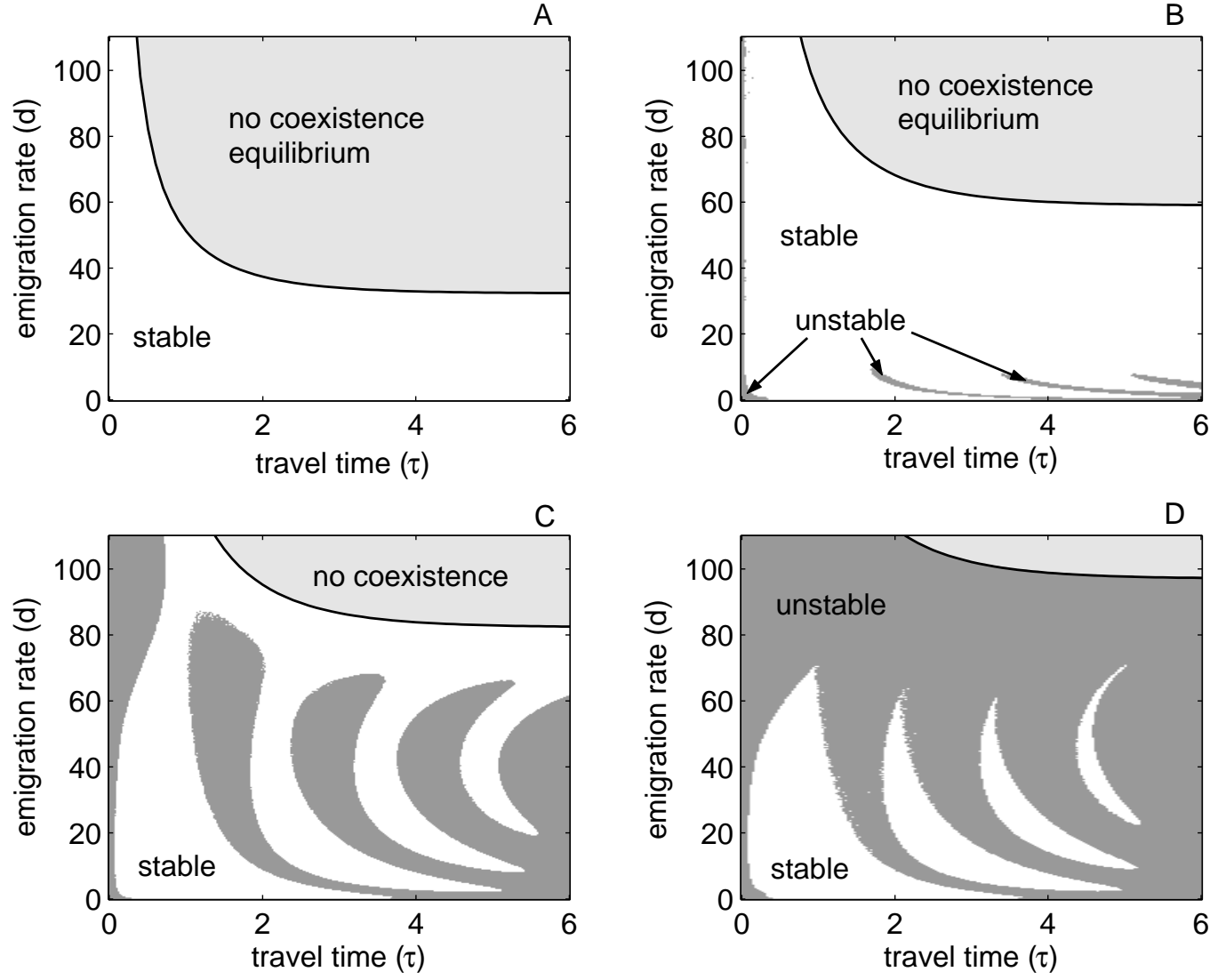

Fig. 1. Stability diagrams from simulations of model (5) with discrete dispersal delay for various values of $\kappa, \varepsilon=0.01, \mu=\mu_{p}=1$ : A) $\kappa=50$; B) $\kappa=150$; C) $\kappa=500$; D) $\kappa=5000$. White areas designate a stable equilibrium point, dark gray stands for an unstable equilibrium point and an area where there is no positive coexistence equilibrium is shown in light gray, bounded by the black curve, i.e., the case of equality (8). 

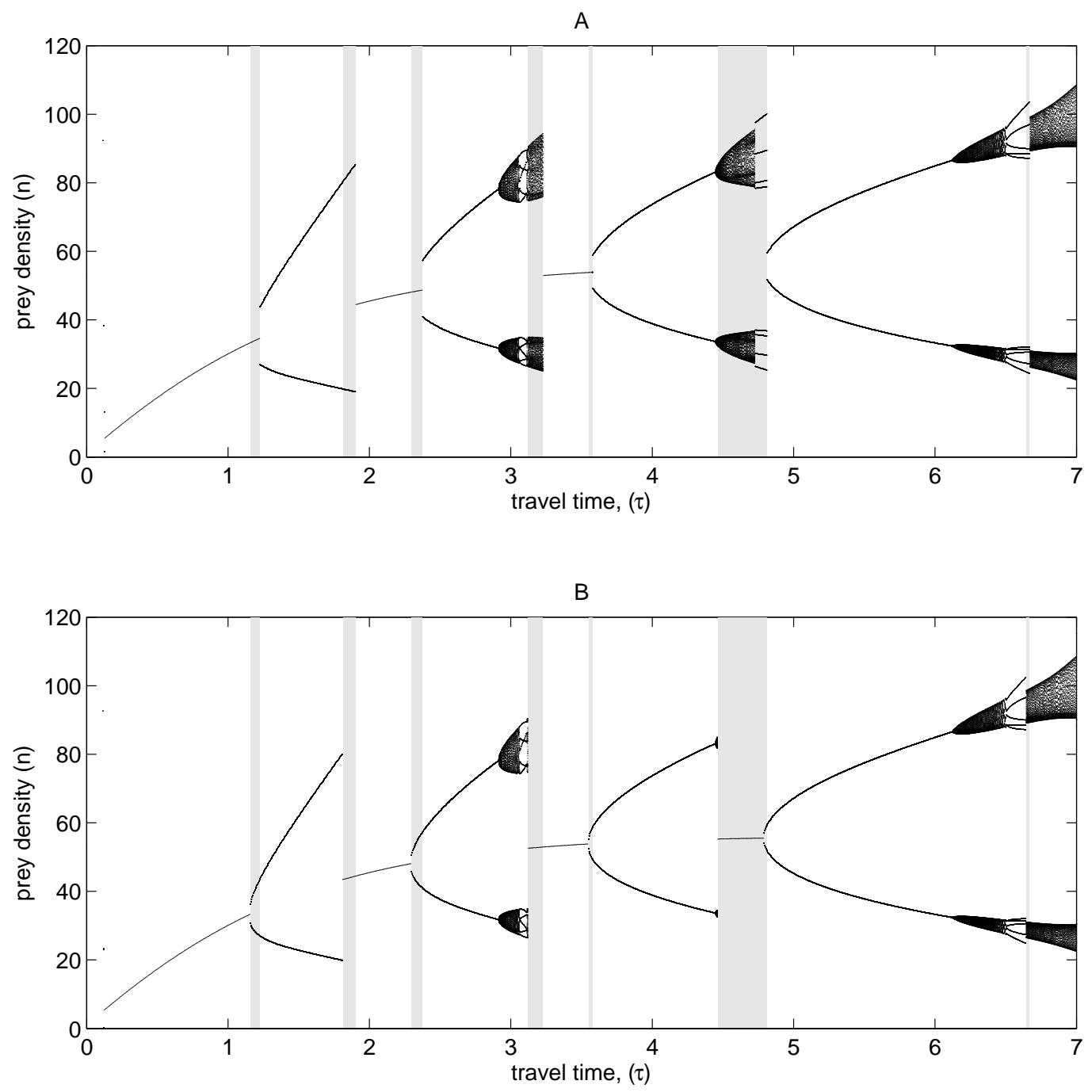

Fig. 2. Bifurcation diagrams for the prey population density of model (5) with discrete dispersal delay (minimum and maximum population densities), $d=35$, $\varepsilon=0.01, \kappa=5000, \mu=\mu_{p}=1$. A) $\tau$ is changed forwards; B) $\tau$ is changed backwards. Shaded regions depict the coexistence of multiple attractors. Detailed explanation in the text. 

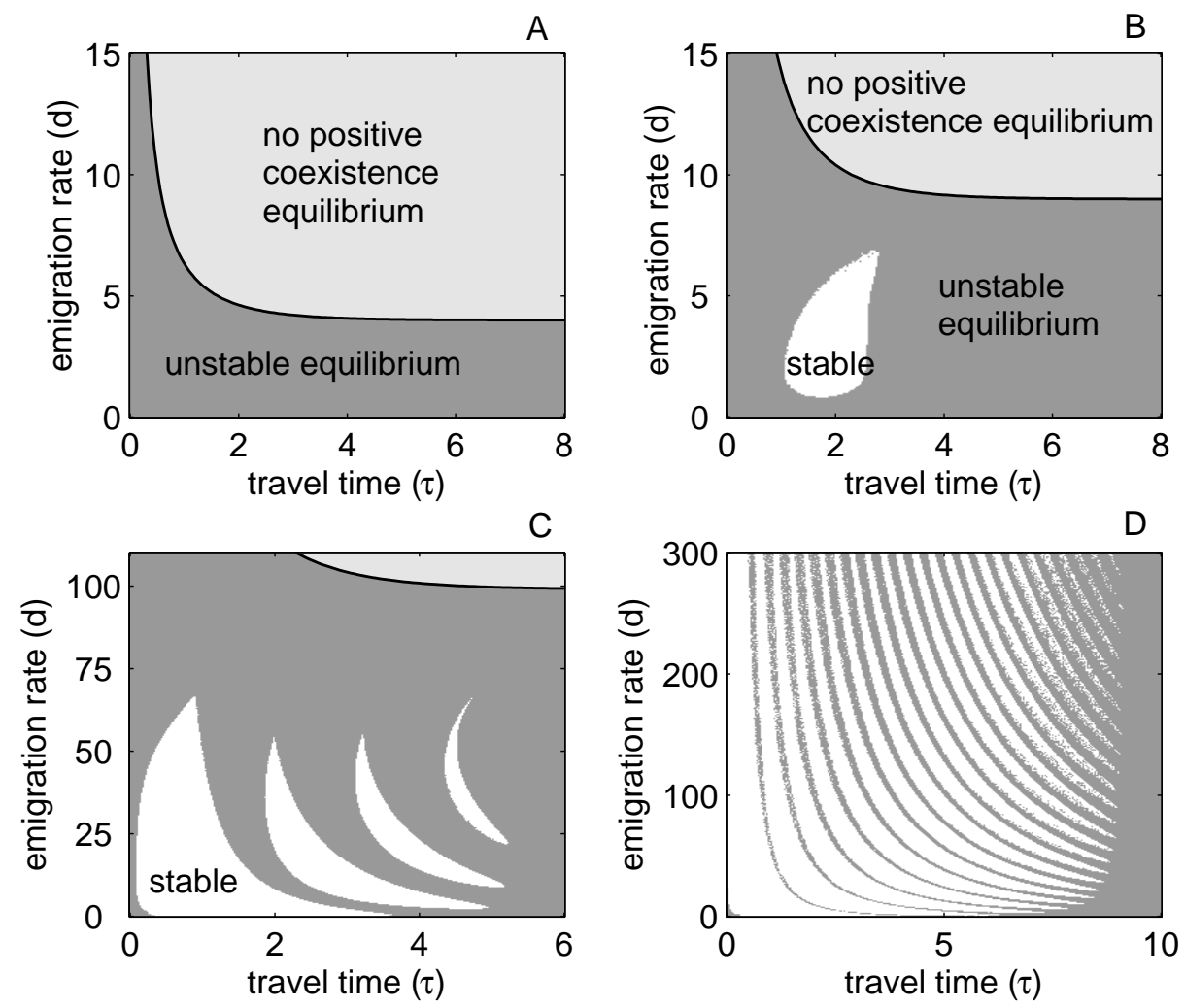

Fig. 3. Stability area of the Type II model (17) with discrete dispersal delay for various $\varepsilon$ : A) $\varepsilon=0.2$; B) $\varepsilon=0.1$; C) $\varepsilon=0.01$; and D) $\varepsilon=0.0002$. Other parameter values are as in Fig. 1. 

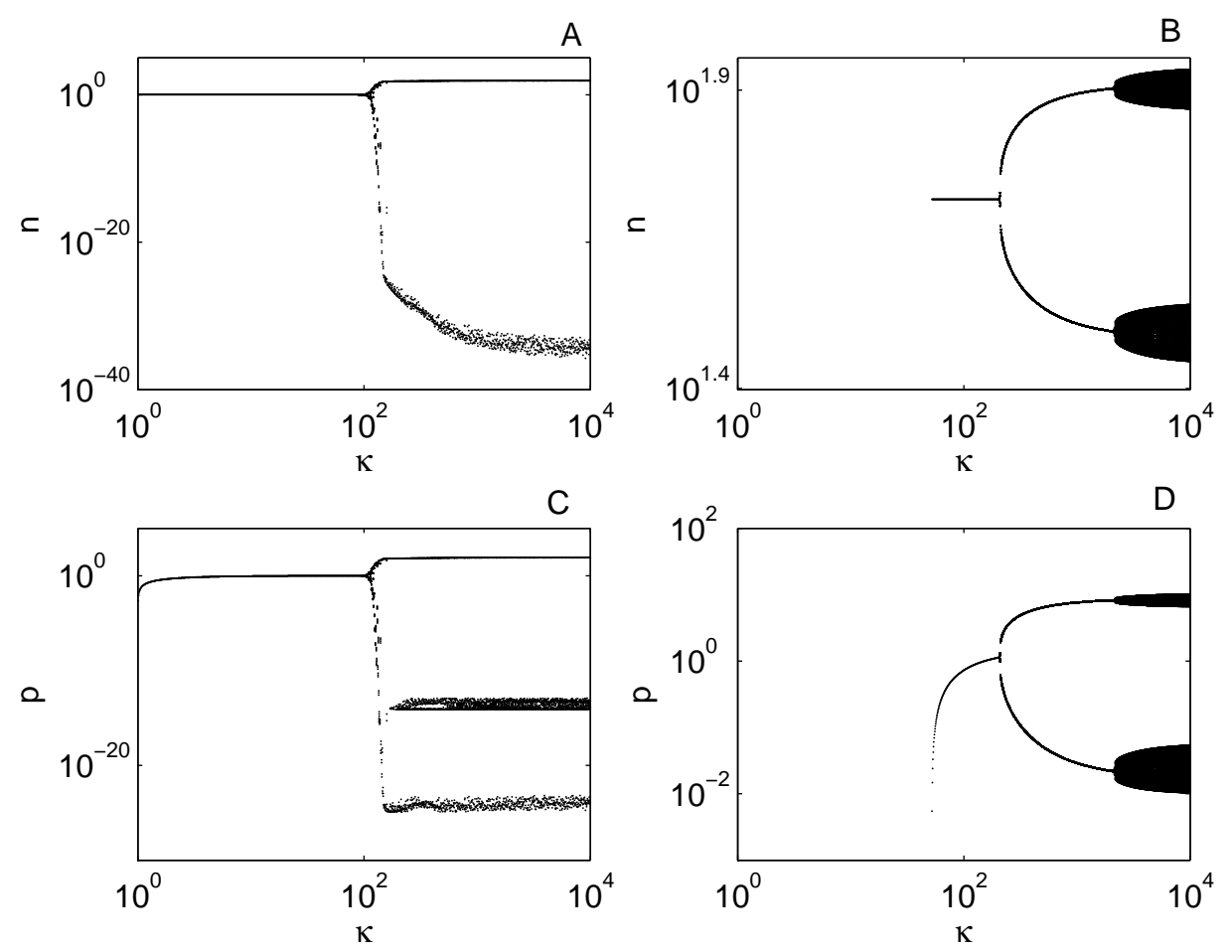

Fig. 4. Bifurcations diagram for model (5) with $\kappa$ as bifurcation parameter show that the amplitude of oscillation is significantly smaller in the presence of dispersal delays; $d=35, \varepsilon=0.01, \mu=\mu_{p}=1$. A) prey population, no dispersal $(\tau=0)$; B) prey population, $\tau=3$; C) predator population, no dispersal $(\tau=0)$; D) predator population, $\tau=3$. 


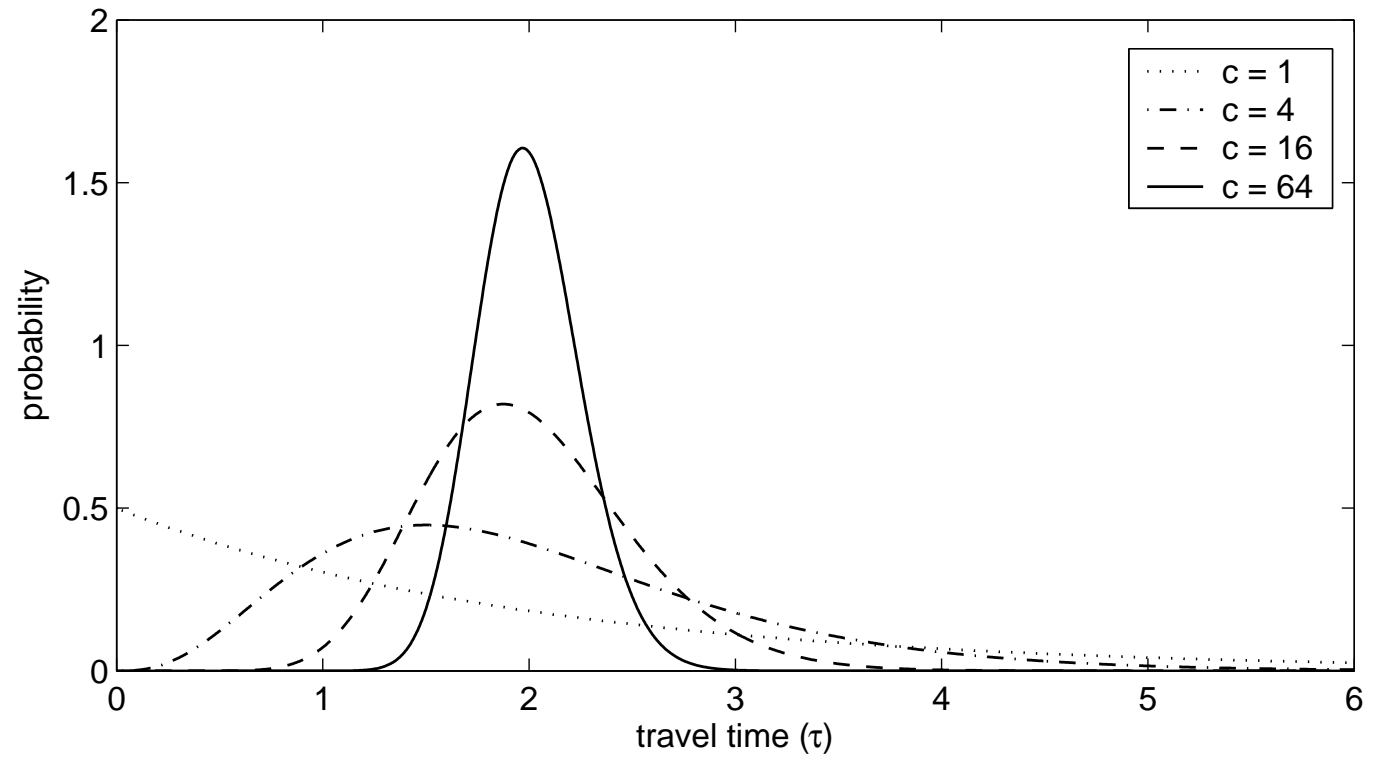

Fig. 5. Shape of the Erlang distribution for increasing values of $c$. The mean of each distribution is fixed at $\tau_{a v}=2$; thus $b=c / 2$ for each curve. Notice that distribution of travel times is narrower for larger $c$. 

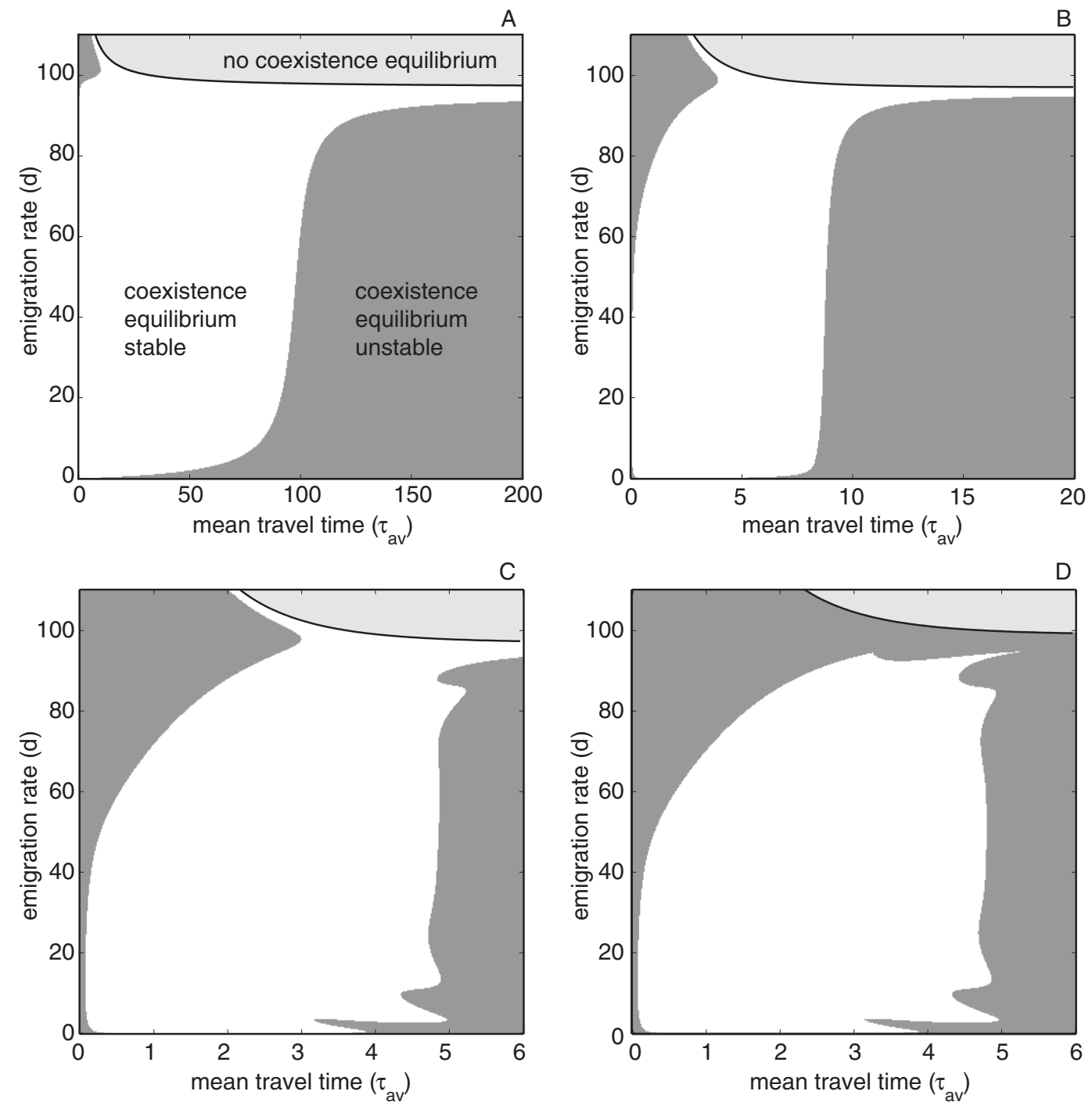

Fig. 6. A-C) Stability diagrams for model (5) with Erlang distributed dispersal-delays computed from $(20) ; \varepsilon=0.01, \mu=\mu_{p}=1, \kappa=1000$. A) $c=1$. B) $c=4$. C) $c=64$. D) Stability diagram for the Type II model (17) with Erlang distributed delay; $c=64$. Note the changing scale of the $\tau_{a v}$-axis. 